\title{
Linking energy system and macroeconomic growth models
}

\author{
Nico Bauer • Ottmar Edenhofer • \\ Socrates Kypreos
}

\begin{abstract}
We compare two alternative approaches for coupling macroeconomic growth models (MGM) and energy system models (ESM). The hard-link approach integrates the techno-economics of the ESM completely into the MGM and solves one highly complex optimisation problem. The soft-link leaves the two models separate and energy supply functions are integrated into the MGM that are derived from the optimal solution of the ESM. The energy supply functions relate the price of energy computed with the ESM to the quantity of energy computed with the MGM. An iterative process exchanges price-quantity information between the models. Hence, the soft-link leads to an energy market equilibrium. But energy supply functions do not consider variable interest rates that influence the energy supply functions. This is due to the fact that ESMs are partial models that assume an exogenous interest rate; however the interest rate is computed endogenously in MGMs. This missing interaction leads to a capital market dis-equilibrium in the soft-link compared to the hard-link approach
\end{abstract}

We would like to thank Leonardo Barreto, Christoph Boehringer, Valentina Bosetti, Elmar Kriegler, Marian Leimbach, Kai Lessmann, Emanuele Massetti, Massimo Tavoni, Hal Turton and Alexander Wokaun for discussions of an earlier draft. Christian Flachsland found many typos and better formulations. All remaining errors are ours.

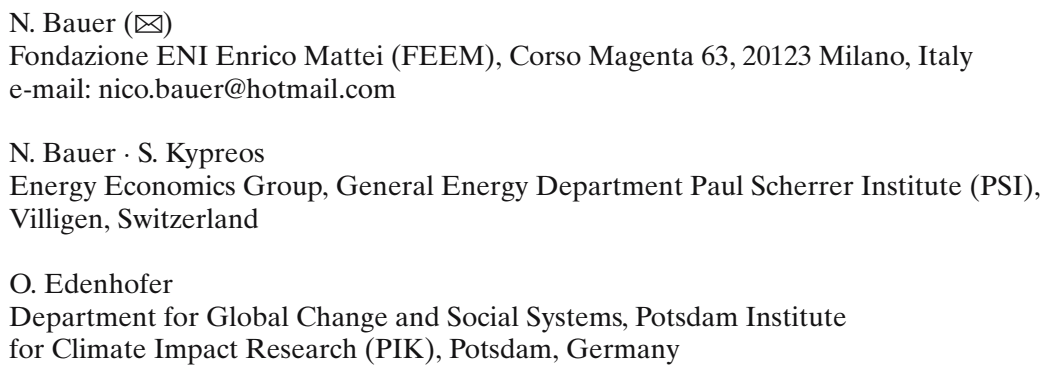


inducing a mis-allocation of investments. Extending the soft-link approach by also considering the time variable interest rate of the MGM does not improve the results. Though the computational complexity is greater the hard-link approach assures simultaneous energy and capital market equilibrium.

Keywords Model coupling - Computational economics - Supply theory · Capital theory · Energy system model · Growth model · Transition dynamics

\section{Introduction}

Analysing the interaction of the macroeconomy and the energy sector is essential for gaining insights into their mid- to long-term development which depends on investment decision that will be made in the coming decades. Energy is an essential production factor for the generation of economic values. This implies that increasing scarcity of energy is an impediment on economic growth and leads to a shift of income distribution favouring energy at the expense of capital and labour. Moreover, econometric analysis finds that energy prices affect the conflict between capital and labour with respect to their income shares; see e.g. Frondel and Schmidt (2004). On the other hand the energy sector requires considerable financial means to install the capacities as well as the related infrastructure with long technical life and pay-back times. Energy related investments are in competition with consumption, investments in other capital stocks and income redistribution within the living generation. The interplay between both sectors affects investments into various long-lived energy technologies, which in turn imply particular primary and end-use energy mixes that are required to generate economic value. Moreover, the energy sector is related to issues of local and global environmental problems, research and development, international trade and public finance.

In the literature several approaches have been introduced to take such interrelationships into account. Apart from some approaches developed in the seventies, the contemporary scientific literature considers five approaches. First, within an energy system model price dependent energy demand represents the flexibility of the economic sector regarding energy savings. Energy price elasticities are usually assumed to be lower than one, which is in correspondence with limited possibilities of substitution. See for example Rafaj (2005) and Smekens and van der Zwaan (2006) and the references therein. However, this approach does not consider the scarcity of sources to finance investments. The second approach models linear energy cost-functions with exogenously assumed coefficients for energy production within a macroeconomic growth model; see e.g. Manne et al. (1995). This approach also ignores the scarcity of capital because the cost-coefficients contain the capital costs as an annuity. The third approach is to formulate a computable general equilibrium model (CGE), where energy conversion technologies are considered as independent economic sectors that interact and compete with the rest of the economy on markets for production factors and output goods; see e.g. Boehringer (1998), Drouet et al. 
(2006) and Wing (2006). Although CGEs consider all of the above interrelationships, they are essentially static if they are solved as recursive dynamic models; i.e. a sequence of static equilibria is intertemporaly connected with parameterised savings. Therefore, the class of CGE models does not consider the issue of the long life-time of energy investments. Fourth and fifth, the so-called soft-link and hard-link approaches are the main subject of this paper and treated next.

The issue of mutual intertemporal interdependency is the motive for integrating macroeconomic growth models (MGM) and energy system models (ESM). The MGM maximises a non-linear intertemporal social welfare function depending on the consumption time path by allocating the budget between consumption and investment. Investments add to the capital stock which produces economic value in combination with labour; the interest rate of capital is determined endogenously. The ESM minimises the discounted costs of the energy system subject to an exogenous energy demand path and the characteristics of energy technologies by choosing investments in capacities. The sources of financial means for investments are not constrained in an ESM and the interest rate is assumed exogenously.

Integration of both models requires - in a first step-energy being considered as a production factor in the MGM. Also the production of energy requires financial means that are accounted in the budget equation of the macroeconomy. In a second step a decision between two approaches of coupling has to be made. First, the hard-link approach integrates the techno-economic constraints of the ESM into the MGM as an additional set of functions and constraints and solves one very complex non-linear programming (NLP) problem. The softlink approach separates the two models and integrates a reduced form model of the ESM into the MGM resulting in a less complex model. The parameters of the reduced form model are calibrated according to the optimal solution of the original ESM that receives information on the exogenous energy demands from the MGM. In an iterative procedure the parameters of the reduced form model are adapted until changes of the energy demand paths between iterations get sufficiently small. The optimal solutions of the ESM and MGM are the solution of the soft-link. The soft-link approach examined in this study has been introduced by Messner and Schrattenholzer (2000). It employs a set of energy supply functions as the reduced form model and their shapes are up-dated between iterations.

The hard-link approach leads to one highly complex NLP problem. This limits the level of disaggregation and detail of both sectors because of the computational abilities of NLP solvers; see e.g. van der Zwaan et al. (2001) and Edenhofer et al. (2005). However, the hard-link computes a consistent solution. The soft-link approach at best reproduces it because there the MGM relies on a reduced form model of the ESM. Thus, the limitations of the soft-link arise from the separate determination of investments paths in the two models that treat capital scarcity differently. However, the ESM and the MGM could comprise more system complexities and still are solvable while the corresponding NLP problem of the hard-link approach could not be solved at all. 
The soft-link computes an equilibrium for the energy market, which confirms internal consistency. However, if we compare energy prices and quantities with those computed with the hard-link, we find considerable deviations. Analysing the appearing differences of the two approaches will point to an inconsistency of the soft-link with respect to capital markets, which in turn has effects on the energy market. The reason is that the endogenous valuation of capital scarcity in the MGM is not considered in the ESM and therefore the supply curves are not consistent with the interest rate. It is a natural step to extend the soft-link approach by also considering the information of the endogenously computed time-variable interest rate of the MGM within the ESM. It turns out that this extension does not improve the soft-link approach, since the distorting effects are not reduced.

We use relatively simple MGM and ESM models and couple them using the hard as well as the soft-link approach. Addressing the consistency of the two coupling approaches justifies the use of simple models. The paper does not make statements on the computational efficiency by comparing computation time with approximation errors. Sensible conclusions on this issue would require more sophisticated models that are calibrated and validated against real world data. Although the motivation for the present study is the coupling of two specific model types, this paper constitutes a contribution to the more general economic issue of supply function in macroeconomic growth models as well as production and capital theory.

The organisation of the paper is as follows. Section 2 presents the two models, while Sect. 3 presents the two coupling approaches in detail and develops the particular research questions on that basis. Section 4 states the results and discusses the questions. The discussion leads to the natural extension of the soft-link approach by considering time variable interest rates in the ESM in Sect. 5. Finally, conclusions are drawn and suggestions for further research are made in Sect. 6.

\section{Two models}

In this section the ESM and MGM models are each introduced in detail. They will then be integrated following the soft- and hard-link approaches presented in the next section. Section 2.1 will introduce the ESM and Sect. 2.2 the MGM.

\subsection{The energy system model}

In general an ESM computes a cost-minimal energy system by choosing additions of energy technology capacities and primary energy utilisation to satisfy a given end-use energy demand. The technologies are described by technical characteristics like conversion efficiencies, emissions, etc., as well as economic characteristics like unit investment costs. Some energy technologies requires scarce primary energy carriers that are described in terms of availability, costs 
etc. The analyst is interested in issues like technology choice, energy mix and costs of policy constraints.

ESMs are partial fix-price models of the energy sector. The energy sector is seen as small relative to the overall economy, which justifies the assumption that changes in its factor demands do not change the factor prices. This assumption is necessary to justify the linearity of cost and production functions that are expressed in linear relationships with fixed coefficients.

The ESM used in this study is a very simple linear programming problem. There are two final energy carriers $E_{j}$, with $j=1,2,{ }^{1}$ that represent electric and non-electric energy, respectively. They can be produced by using either scarce fossil fuel or renewable energy sources that are in the following indexed with $F$ and $R$, respectively. Therefore, we have the following balance equation:

$$
E_{j}=\sum_{k=F, R} E_{j k}, \quad \text { for } j=1,2 \text {. }
$$

The production of end-use energy requires capacities that have to be installed. The availability of capacities requires fixed operation and maintenance $(\mathrm{O} \& \mathrm{M})$ efforts, production of energy by utilising the capacities requires variable $\mathrm{O} \& \mathrm{M}$ efforts and if $k=F$ fossil fuels according to the conversion efficiencies. Thus, there are three cost components of the total costs $C_{t o t}$ in each period: investment costs $C_{I}$, fixed and variable O\&M costs $C_{O M}$ and fossil fuel costs $C_{F}$ :

$$
C_{t o t}=C_{I}+C_{O M}+C_{F} .
$$

The capacities $K^{E}$ are required for the production of useful energy. The availability of a capacity during a year is the load factor $v$, which is assumed exogenously. Thus, the energy production per year is

$$
E_{j k}=v_{j k} K_{j k}^{E}, \quad \text { for } j=1,2 \text { and } k=F, R \text {. }
$$

Addition of energy conversion capacities $I^{E}$ leads to investment costs that are proportional according to the investment costs per unit $\iota$ :

$$
C_{I}=\sum_{j=1,2} \sum_{k=F, R} \iota_{j k} I_{j k}^{E} .
$$

$K^{E}$ increase with capacity additions $I^{E}$ and decline exponentially according to the depreciation rate $\delta$; the initial conditions are $K^{E, 0}$ :

$$
\dot{K}_{j k}^{E}=I_{j k}^{E}-\delta_{j k} K_{j k}^{E}, \quad K_{j k}^{E}\left(t=\tau_{1}\right)=K_{j k}^{E, 0}, \quad \text { for } j=1,2 \text { and } k=F, R
$$

The fixed O\&M costs are proportional to the capacities using the cost coefficient $o^{f}$ and the variable O\&M costs are proportional to energy production

\footnotetext{
1 In the following we skip the indices of parameters and variables, if they are redundant.
} 
using the cost coefficient $o^{v}$ :

$$
C_{O M}=\sum_{j=1,2} \sum_{k=F, R} o_{j k}^{f} K_{j k}^{E}+o_{j k}^{v} E_{j k} .
$$

The fossil fuel costs $C_{F}$ are determined by the fossil resource extraction $R_{l F}$ and the producer costs of fossil fuels $p_{l F}$ :

$$
C_{F}=\sum_{l} p_{l F} R_{l F}
$$

The index $l$ distinguishes the various deposits of fossil fuels that are characterised by $p_{l F}$ and the maximum available amount $R_{l F}^{\max }$. This means that there are deposits with relatively cheap extraction costs being exhausted first, afterwards the next cheapest deposit is exhausted and so forth. The overall amount of all deposits is assumed sufficiently high, so that the overall energy demand could be satisfied from this source. Fuel extraction from all deposits has to cover the total fuel demand that is linearly related to $E_{j}$ by the conversion efficiencies $\eta$ :

$$
\sum_{l} R_{l F}=\sum_{j=1,2} \frac{1}{\eta_{j F}} E_{j F}
$$

The maximally available amount from each deposit leads to $l$ intertemporal resource constraints:

$$
R_{l F}^{\max } \geq \int_{\tau_{1}}^{\tau_{2}} R_{l F}(t) d t, \quad \forall l .
$$

For the remainder of the paper it is useful to term Eqs. 1-9 the techno-economic constraints of the ESM.

The ESM can be run in a stand alone version. For this it is necessary to consider a demand constraint, which requires that exogenously given energy demands $\bar{E}_{j}$ need to be satisfied by the energy production $E_{j}$. Moreover, we introduce a criterion that shall be optimised. Thus, the optimal control problem is to minimise the cumulative discounted energy system costs DESC by choosing capacity additions $I^{E}$ subject to the demand scenario $\bar{E}$ and the techno-economic constraints. For discounting we assume exogenously a constant interest rate $r$. The resulting ESM is denoted $\mathcal{E}$ :

$$
\begin{aligned}
\operatorname{Min}_{I^{E} \geq 0} D E S C & =\int_{\tau_{1}}^{\tau_{2}} e^{-r t} C_{t o t}(t) d t ; \\
\text { s.t. } \bar{E}_{j}(t) \leq & E_{j}(t), \quad \text { for } j=1,2 \\
& \text { Eqs. } 1-9 .
\end{aligned}
$$


The objective function in Eq. 10 implicitly assumes that the energy sector can get as much financial means as it demands at any time at a constant interest rate $r$. The demand constraint in Eq. 11 implies a price inelastic energy demand.

\subsection{The macroeconomic growth model}

A simple Ramsey-type MGM - for the time being considered without energy computes a welfare optimal growth path of a total economy by allocating economic income to investments and consumption. The economy is described by non-linear welfare and production functions, an accounting system of the entire economy, a capital motion equation with an initial capital stock and exogenous development of labour. The analyst is interested in welfare optimal transition and steady-state paths of investment rates, interest rates, distribution of income and economic growth.

An MGM is a general flex-price model of a whole economy. The accounting system is consistent: all expenditures are incomes and all incomes are used for alternative purposes. Thus, the total economic output is distributed among the production factors and the consumption and investment expenditures can not exceed the economic income. Balancing the consumption-investment decision in the light of limited income requires flexible prices represented in the non-linear production and utility functions.

The MGM in this study is an extended Ramsey-model that considers the production factor energy explicitly. It solves the consumption-investment-energy expenditure decision by maximising intertemporal social welfare $W$, that is computed as follows:

$$
\begin{aligned}
W=\int_{\tau_{1}}^{\tau_{2}} e^{-\rho t} U(C(t)) d t, \quad \text { with } \quad W & \in \mathbb{R} \text { and } U(\cdot) \in \mathbb{C}^{2} ; \\
U^{\prime} & >0, U^{\prime \prime}<0 ; \lim _{c \rightarrow 0} U^{\prime} \rightarrow \infty, \lim _{c \rightarrow \infty} U^{\prime} \rightarrow 0 .
\end{aligned}
$$

$W$ is the scalar welfare measure that is computed as the discounted sum over the time interval $\left[\tau_{1}, \tau_{2}\right]$ of the utility path; $\rho$ is the discounting rate. Utility at a time is computed using the utility function $U(\cdot)$, which is a iso-elastic function in consumption $C \geq 0$.

$C$ is the residual of economic income $Y$ that is alternatively allocated to capital investment $I^{M}$ or to energy related expenditures $E E$ :

$$
Y=C+I^{M}+E E .
$$

$I^{M}$ is allocated to two capital stocks $\left(K_{A}^{M}, K_{B}^{M}\right)$ representing the industry and the transport sector. The stocks are subject to depreciation and the initial condition of historically given capital stocks $K_{i}^{M, 0}$ : 


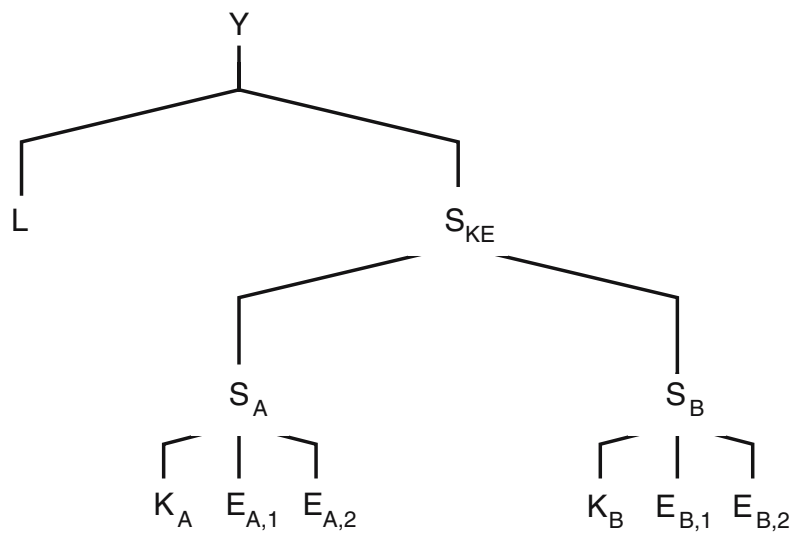

Fig. 1 Production structure of the nested CES given in Eqs. 16-17

$$
\dot{K}_{i}^{M}=I_{i}^{M}-\delta_{i} K_{i}^{M}, \quad K_{i}^{M}\left(t=\tau_{1}\right)=K_{i}^{M, 0}, \quad \text { for } i=A, B .
$$

The capital stocks are required for the production of economic values. Each capital stock is combined with two types of energy $E_{1}$ and $E_{2}$ in order to form sectoral composites $S_{A}$ and $S_{B}$. The two composites are again combined to an aggregate capital-energy composite $S_{K E}$ that in turn is combined with exogenously assumed labour $L$ in order to compute the produced economic value $Y$. The general form of this production structure is

$$
Y=F\left\{A \cdot L, S_{K E}\left[S_{A}\left(K_{A}^{M}, E_{A 1}, E_{A 2}\right), S_{B}\left(K_{B}^{M}, E_{B 1}, E_{B 2}\right)\right]\right\} .
$$

Applying the concept of constant elasticity of substitution (CES) production functions results in the CES-nesting structure shown in Fig. 1 and more formally to the following equations:

$$
\begin{aligned}
Y=F\left(A \cdot L, S_{K E}\right) & =\left[\tilde{\xi}_{L}(A \cdot L)^{\tilde{\sigma}_{S_{K L E}}}+\tilde{\xi}_{S_{K E}} S_{K E}^{\tilde{\sigma}_{S_{K L E}}}\right]^{\frac{1}{\tilde{\sigma}_{S L E}}} ; \\
\text { with } S_{K E}\left(S_{A}, S_{B}\right) & =\left[\sum_{i=A, B} \tilde{\xi}_{S_{i}} S_{i}^{\tilde{\sigma}_{S_{K E}}}\right]^{\frac{1}{\tilde{\sigma}_{S E}}} ; \\
\text { with } S_{i}\left(K_{i}^{M}, E_{i j}\right) & =\left[\tilde{\xi}_{K, i} K_{i}^{M^{\tilde{\sigma}_{S}}}+\sum_{j=1,2} \tilde{\xi}_{E, i j} E_{i j}^{\tilde{\sigma}{ }_{S i}}\right]^{\frac{1}{\tilde{\sigma}}}, \quad \text { for } i=A, B .
\end{aligned}
$$

In these equations $\xi$ and $\tilde{\sigma}$ denote the parameters for distribution and substitution, respectively, as they are common in CES production functions; see e.g. Arrow et al. (1961). The common definition is applied for the elasticity of 
substitution $\sigma=(\tilde{\sigma}-1)^{-1}$. The parameter $A$ is a factor that represents the efficiency level of labour and it increases with time.

Energy of both types is delivered from the energy sector as will be described in the next section. For both types of end-use energy $E_{j}$ the following balance equations have to be fulfilled:

$$
E_{j}=\sum_{i=A, B} E_{i j}, \quad \text { for } j=1,2 .
$$

The model is not complete, yet. It lacks of the relationship that maps time paths of $E_{j}$, with $j=1,2$ into time paths of $E E$. This relationship depends on the coupling approach that links it with the ESM and is explained in the next section.

The use of disaggregate capital stocks in a nested CES production function is new in this field of applied modelling. Although the capital stocks are measured in the same units, they could not be aggregated, since the elasticities of substitution $\sigma_{S_{A}}$ and $\sigma_{S_{B}}$ are not equal. Bauer (2005, Chap. 3) provides a detailed discussion of the conditions of capital stock aggregation in nested CES production functions.

\section{Two approaches}

In this section the two approaches for coupling MGM and ESM are introduced in detail. Section 3.1 introduces the hard-link approach and Sect. 3.2 deals with the soft-link approach. In Sect. 3.3 we add some remarks and motivate the research questions of this study.

\subsection{The hard-link approach}

The hard-link approach integrates the techno-economic constraints of the ESM into the MGM; we term the resulting model $\mathcal{M}_{H L}$. In particular, Eqs. 1-9 become additional constraints in the MGM and the energy related expenditures $E E$ equal the total energy system costs $C_{t o t}$ of Eq. 2. Therefore, the budget equation in the hard-linked model is

$$
Y=C+I^{M}+C_{t o t} .
$$

The objective function in Eq. 10 and demand constraint in Eq. 11 are obsolete in the hard-link approach, since the one and only optimisation problem is to maximise $W$ of the MGM given in Eq. 12 and energy demand is endogenous. The hard-link approach optimises welfare $W$ by choosing investments $\left(I^{M}, I^{E}\right)$ subject to the macro-economic constraints and the techno-economic constraints of the ESM in one optimisation problem. Formally, the optimal control problem of $\mathcal{M}_{H L}$ is: 


$$
\begin{aligned}
\underset{I^{M}, I^{E} \geq 0}{\max } & =W ; \\
\text { s.t. } & \text { Eqs. } 1-9, \\
& \text { Eqs. } 14-20 .
\end{aligned}
$$

The hard-link approach considers all available information in an integrated framework that is solved simultaneously. The optimal solution of $\mathcal{M}_{H L}$ comprises investment paths $I^{E}$ and $I^{M}$ and is interpreted to be the best solution available. The soft-link works differently, since $I^{E}$ and $I^{M}$ are determined in two models that are not integrated but related through energy supply functions. Thus, to study the appropriateness of the soft-link approach the solution of $\mathcal{M}_{H L}$ is set as the benchmark to compare it to the outcome of the soft-link approach that is introduced next.

\subsection{The soft-link approach}

Formalising the soft-link approach using the two models of Chap. 2 requires four steps:

1. A reduced form model of the ESM has to be formulated representing the full scale ESM and needs to be integrated into the MGM.

2. Parameter determination of the reduced form model taken from the optimal solution of the $\mathcal{E}$.

3. An iteration procedure that defines the succession of computations and the information flow between the models is introduced.

4. A stopping criterion has to be defined in order to stop the iteration.

The first step in formulating the soft-link approach is to build a reduced form model of the ESM, which is then integrated into the MGM. This model is denoted $\mathcal{M}_{S L}$. A commonly used reduced form model follows the supply curve concept: the energy related expenditures EE in Eq. 13 depend on the amount of the two types of energy delivered to the macroeconomic production function:

$$
E E(t)=a(t)+\sum_{j=1,2} b_{j}(t) E_{j}(t)^{c} .
$$

Thus, the model $\mathcal{M}_{S L}$ has the following form:

$$
\begin{aligned}
\underset{I^{M}, E \geq 0}{\operatorname{Max}} & =W ; \\
\text { s.t. } & \text { Eqs. 13, 22, } \\
& \text { Eqs. } 12,14-19 .
\end{aligned}
$$

The model $\mathcal{M}_{S L}$ solves for the time paths of $I^{M}$ and $E$. It is different from $\mathcal{M}_{H L}$ in that it does not control energy investments $I^{E}$. These are determined in the model $\mathcal{E}$ and considered only indirectly via Eq. 22. 
In the second step the time paths of the parameters $a$ and $b=\left(b_{1}, b_{2}\right)$ are derived from the optimal solution of $\mathcal{E}$ for a set of exogenously given energy demand paths $\bar{E}=\left(\bar{E}_{1}, \bar{E}_{2}\right)$ in Eq. 11 . The parameter $c$ is assumed to equal two. Hence, $E E$ is convex in $E$, which presupposes a production function with diminishing returns to scale.

The parameters $b$ are determined by the following equation that is derived from the partial derivative of Eq. 22 with respect to $\bar{E}_{j}$, for $j=1,2$. The partial derivative is assumed to equal the price of energy, which is assumed to be the shadow price of energy type $j$ denoted $\mathcal{P}_{j}$. It is the dual variable of the energy demand constraint Eq. 11 of the optimal solution of $\mathcal{E}$ in each time step:

$$
b_{j}=\frac{\mathcal{P}_{j}}{c \cdot \bar{E}_{j}^{c-1}}, \quad \text { for } j=1,2 .
$$

The parameter $a$ is interpreted as a technically necessary correction parameter. The variable energy costs $\sum_{j} b_{j} \bar{E}_{j}^{c}$ might not equal the total energy system costs $C_{t o t}$. Thus, the fixed energy costs $a$ are introduced to correct for this difference:

$$
a=C_{t o t}-\sum_{j=1,2} b_{j} \bar{E}_{j}^{c}
$$

Defining in the third step the iteration makes use of step one and two. Additionally considering Eqs. 24 and 25, $\mathcal{E}$ can be interpreted as a function mapping time paths of $\bar{E}$ into time paths of $a$ and $b$ :

$$
\left(\begin{array}{l}
a \\
b
\end{array}\right)=\mathcal{E}(\bar{E})
$$

In turn, $\mathcal{M}_{S L}$ can be interpreted as a function mapping the supply curve parameters $a$ and $b$ into energy demand paths $\bar{E}$

$$
\bar{E}=\mathcal{M}_{S L}(a, b) .
$$

The mappings Eqs. 26 and 27 define an iteration procedure to find a fixed point for $\bar{E}$, since the input of one model is the output the other. Thus, choosing an arbritray initial path for $\bar{E}$, and solving Eq. 26 we get the demand curve parameters, that are used to solve Eq. 27, which results in a new time path for $\bar{E}$; this closes the iteration loop.

As a stopping criterion one can ask in the final step for less than $\epsilon$-changes of $\bar{E}$ from one to the next iteration. The solution is found as soon as the iteration stops due to this convergence criterion.

The result of the soft-link approach comprises (i) paths for $I^{M}$ and $E$ determined in the model $\mathcal{M}_{S L}$ depending on the supply function parameters and (ii) paths for $I^{E}$ determined in the model $\mathcal{E}$ depending on $\bar{E}$. Hence, the investments $I^{M}$ and $I^{E}$ are determined in two separate models. For the remainder of the 
paper it should be kept in mind that the MGM determines the interest rate endogenously, while the ESM assumes it exogenously.

\subsection{Remarks and research questions}

We make some remarks regarding the two concepts and relate them to the four research questions. First, the iteration in the soft-link approach stops as soon as the changes of the energy demand paths $\bar{E}$ become sufficiently small. The algorithm does not look for energy market equilibria nor is it implicitly guaranteed. This leads to the first question: are the supply and demand prices equal?

Second, the parameter $a$ in Eq. 25 closes the gap between the variable costs of the energy system and the actual energy costs. The parameter $a$ could be interpreted as a lump-sum tax transfer to the energy sector, since these are expenses without delivery of energy. This leads to the second question: Is the lump-sum transfer sufficiently small compared to the total energy system costs?

Third, any reduced form model is subject to the question, whether it reproduces the original model sufficiently close and whether it still comprises all essential effects. This points to the consistency of the soft-link with the hard-link approach. We assume the solution of the hard-link approach as the benchmark and ask: What is the difference of energy prices and quantities in the soft-link solution relative to the hard-link solution?

Fourth, the emerging differences of energy prices and quantities computed with the two approaches give rise to the analysis of the treatment of capital scarcity in both approaches. The focus is moved to the treatment of capital markets and how investments are allocated in both approaches. Hence, the fourth research question is: What is the role of the capital market in the soft and the hard-link approach and how does it affect the results?

The next two remarks are with respect to the static nature of (energy) supply functions. Every supply function assumes that all rewards and expenses are realised during the same period in which the production takes place. The ESM is essentially different in this respect, since the expansion of supply in one period induces the expansion of supply in future periods because the capacities are installed. To put it differently: The expansion of supply requires financial means for investments that have a payback period that is much longer than the period for which the supply increase is considered in a supply function. The supply curve concept assumes full flexibility of quantity choices between periods.

Finally, from this observation the problem of determining the parameters of the supply function arises. One should note that the supply curves are calibrated as convex functions using only one single price-quantity point for each type of end-use energy. An alternative procedure is to compute several price-quantity combinations using the ESM by varying the time paths of $E$ at all time steps. But since a perturbation in $t$ has consequences in all $t+\tilde{t}$, with $\tilde{t} \geq 1$, the supply curve in each period can not be properly determined. The fundamental reason 
is that the supply curve concept simply aims at summarising an essentially dynamic formal structure into a static framework.

\section{Results}

The following computations are performed using arbitrary numbers for the calibration. It is not intended to use the computations to show the effect for any particular country or region. Section 4.1 asks for the internal consistency of the soft-link approach and addresses the first two research questions. In Sect. 4.2 we examine the consistency of the soft- with the hard-link approach and we treat the third and fourth research question.

\subsection{Consistency of the soft-link approach}

The soft-link approach does not guarantee that the supply and demand prices equalise. The demand and supply quantities are equal, since the energy demand of $\mathcal{M}_{S L}$ is the boundary condition for $\mathcal{E}$. The question related to the energy prices does only touch the internal consistency of the soft-link approach.

Figure 2 compares the demand and supply prices of energy that are computed with the soft-link approach. The supply price of a type of energy is determined by the slope of the energy supply curve. The demand price equals the shadow price of an end-use energy type $E_{j}, j=1,2$, using Eq. 19 of the $\mathcal{M}_{S L}$.

Figure 2 a shows the time path of prices for $E_{1}$ as measured on the left hand scale. There is a wedge between both with a slightly higher supply price. The increase of the price is due to the fact that the production of $E_{1}$ relies on the use of the scarce fossil energy sources, which induces a scarcity rent. There is also a small, constant price wedge observed for $E_{2}$ in Fig. 2b; the constancy of the prices is implied by the use of infinite renewable energy sources.

(a)

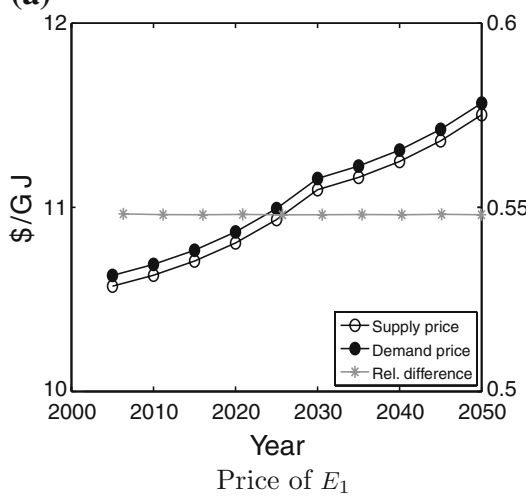

(b)

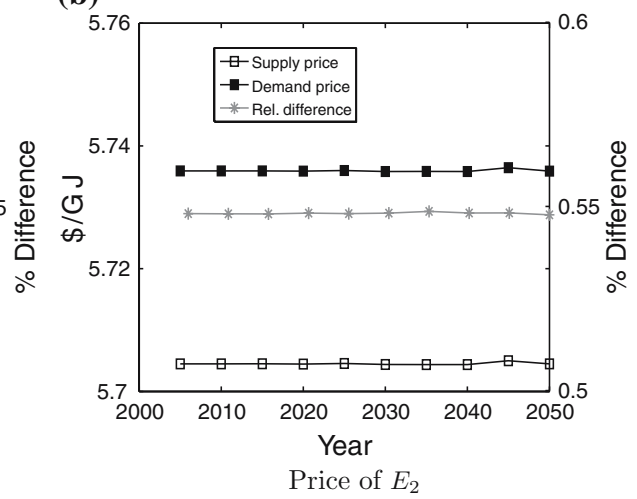

Fig. 2 Time path of demand and supply prices of energy $E_{j}$ in the soft-link computed with $\mathcal{M}_{S L}$ and $\mathcal{E}$, respectively, is shown on the left axis; the relative differences are given on the right axis 
(a)

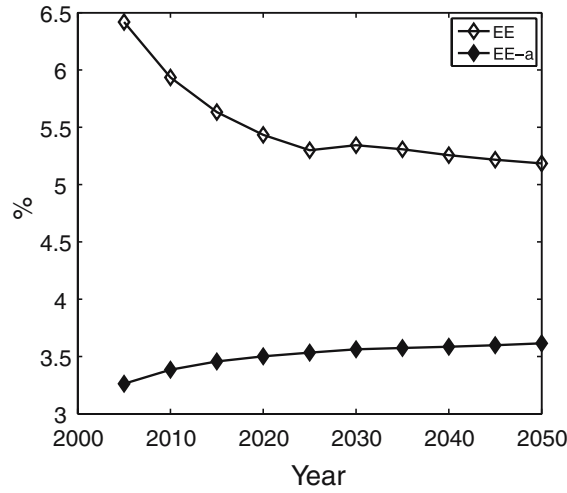

Share of energy system costs (b)

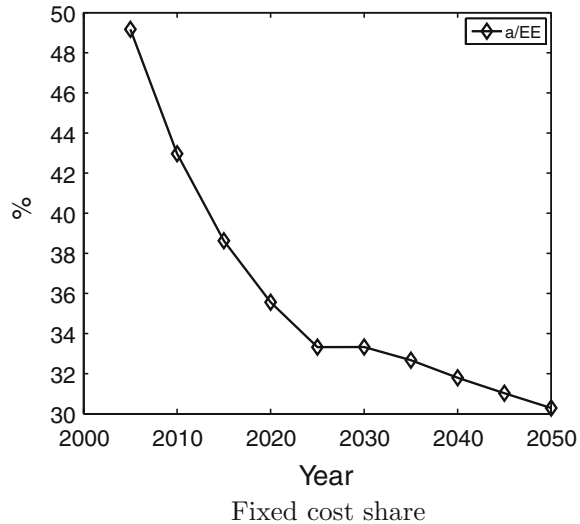

Fig. 3 Time path of the energy system cost computed with the $\mathcal{E}$ and the $\mathcal{M}_{S L}$

The differences are sufficiently small; for both energy types it is about $0.5 \%$ as measured on the right hand scale. Hence, we conclude that the soft-link approach performs well with respect to the equilibrium on the energy market.

We turn to the second research question, which addresses the consistency of expenditures to the energy sector. Although prices and quantities are equivalent for both models, there is no guarantee that the energy expenditures in each period computed with $\mathcal{E}$ are the same as those computed with $\mathcal{M}_{S L}$ using the supply curve. A possible gap is closed by the technical correction factor $a$ and can be interpreted as a lump sum transfer from the household to the energy sector. The question is whether this transfer is significant or negligible.

Figure 3 illustrates the model behaviour. Figure 3 a shows the share of the total $E E$ and the variable energy system costs $(E E-a)$ computed with $\mathcal{M}_{S L}$ relative to macroeconomic output. The time paths show qualitative and quantitative differences. Initially, the share of the total energy system costs is decreasing while the variable energy system costs increase slightly. In the long-run there is a difference of about $1.5 \%$-points, while the absolute share of variable energy system costs is only slightly higher than $3.5 \%$.

Figure $3 b$ shows the share of the fixed costs $a$ relative to the total energy system costs $E E$. In the initial period nearly half of the energy system costs computed with the supply curve are financed by the lump-sum transfer. This share decreases, but remains between 30 and $35 \%$.

The reason for this model behaviour is related to the particular form of the energy supply functions. The quadratic form of Eq. 22 used in this study implies that the first unit of energy is produced at costs equal to zero. However, the energy production costs of any unit of energy computed with $\mathcal{E}$ are higher than zero. The resulting gap in energy costs is closed by the introduction of $a$. Hence, the energy supply curves - each calibrated for one particular price-quantity combination-are only valid in the close neighbourhood of the calibration point. Their sole purpose is defined within the soft-link approach. 
We performed a sensitivity analysis with respect to the parameter $c$ that determines the curvature of the supply functions. The relative differences of energy prices as shown in Fig. 2 do not change. The fixed cost share increases with the parameter and vice versa.

In summary, we conclude that the soft-link approach leads to an internally consistent solution with respect to prices and quantities on the energy market. However, variable energy costs in the soft-link approach-i.e. those justified by economic exchange-deviate substantially. The deviation is addressed by introducing the lump sum transfer $a$. Therefore, the soft-link approach seems useful, if one is interested in a solution assuring energy market equilibrium. The use of the calibrated energy supply curves should be limited to this purpose because the lump sum transfer is quite large.

So far, the analysis does not reveal whether the soft-link approach leads to systematic biases with respect to energy prices and quantities compared to the hard-link approach. This question is addressed next and it turns out that the deviations are substantial.

\subsection{Consistency with the hard-link approach}

In the following we assess the consistency of the soft-link with the hard-link approach by comparing them. The latter is the benchmark that should be reproduced by the former.

The subsection addresses the third and the fourth research question. Addressing the third research question we compare results for energy prices and quantities computed with the two approaches. Explaining the remarkable differences of the results leads us to the fourth research question and we examine the investment dynamics of an economy in transition towards the steady-state growth path. The explanation for the differences requires consideration of the scarcity of capital and allocation of investments - i.e. the intertemporal valuation of investments and consumption - that are treated differently in the two approaches. This will lead us (Sect. 5) to augment the soft-link approach so that the time variable scarcity of capital is also considered in the ESM of the soft-link.

The differences of energy quantities and prices for the soft-link relative to the hard-link are shown in Fig. 4. For both types of energy the traded quantity shows a clear pattern in Fig. 4a. During early periods there is remarkably higher energy production in the soft-link approach, which inverts into lower energy production in later periods. This pattern is mirrored in the time paths of the relative differences of the prices as shown in Fig. 4b. Energy prices in the soft-link approach are initially lower because of the higher energy production; then the difference is reversed because of the lower energy production in the soft-link. The differences are more accentuated for $E_{2}$, which is produced by renewable energy technologies that only require capital as input. Please note that the relative price differences $(-15$ to $15 \%)$ are more pronounced than the relative differences of quantities ( -4 to $2 \%$ ) due to the assumed poor possibilities to substitute energy with capital. 
(a)

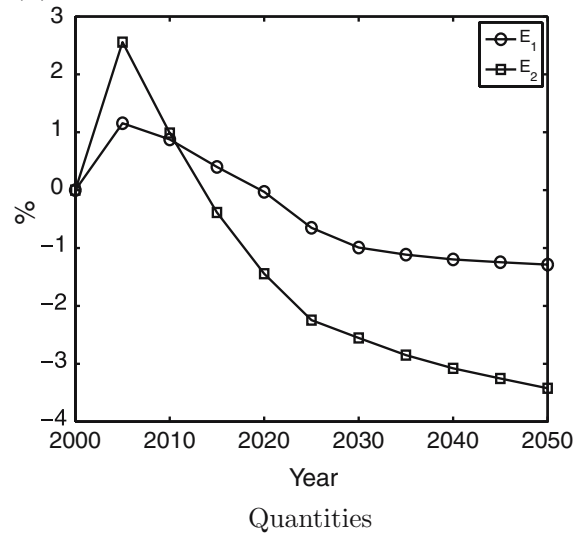

(b)

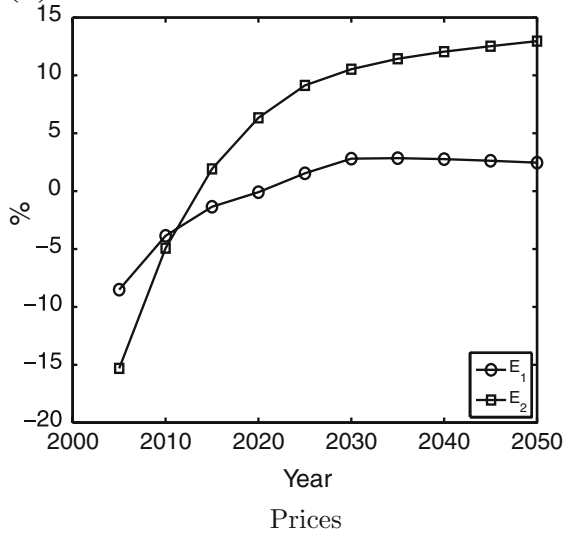

Fig. 4 Differences of the soft-link relative to the hard-link approach for energy quantities and prices

We observe that the time paths of the relative quantity differences are not monotonous in time. In the beginning the soft-link approach shows a greater flexibility in increasing the supply of energy, but in the long-run the energy production capacities are not expanded as much as in the hard-link approach. These non-monotonous differences point into the direction that the soft and the hard-link approach have different dynamic properties related to the accumulation of capacities in the energy sector. This issue is in turn related to the scarcity of capital and the intertemporal valuation of consumption and investment, since these are the control variables. Hence, the soft-link approach using the static supply curve deals with the scarcity of capital in a different way than does the hard-link integrating the overall dynamics of the energy sector investments. This is the subject of the fourth research question and treated next.

Analysing capital market equilibrium requires studying the own rates of return (ORR) of capital and capacity stocks, which are the indicators for the amount and allocation of investments of the economy; see e.g. Dorfman et al. (1958, Chap. 12). ORR are specific to distinct investment opportunities $r_{i^{\prime}}$; in our case the index $i^{\prime}$ distinguishes investments that increase the capital and capacity stocks. The various $r_{i^{\prime}}$ are related to the economy's interest rate $r^{*}$.

The ORR of a technology is the return in monetary terms that could be paid by the investor to the financier for lending the financial means; i.e. credits, stocks, etc. The investors know their ORR and ask for credits at the capital market. If the ORR of a project is higher than $r^{*}$ the investors will demand as much credits as the investment opportunity can pay off. Increased investments into a technology increase the future production of the corresponding good, which leads to lower demand prices and therefore to a lower profitability. Furthermore, increasing investments would increase the market interest rate since the households supply of financial means is trading off with immediate consumption. Hence, investments are not extended to infinity. If $r_{i^{\prime}}$ of a technology 


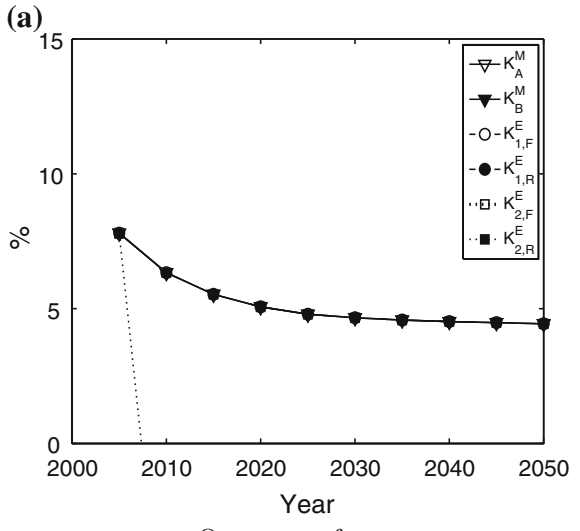

Own rate of return (b)

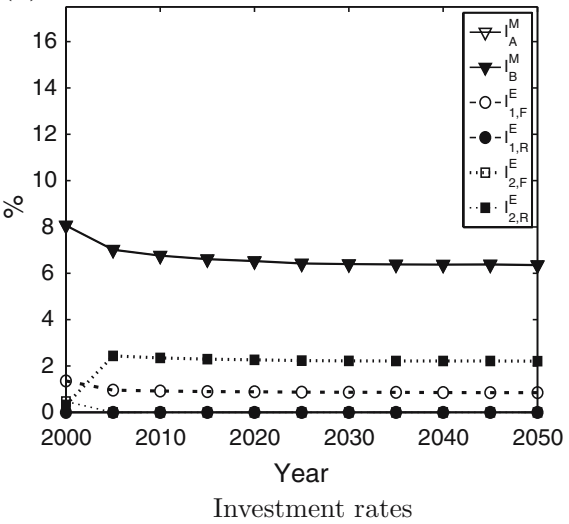

Fig. 5 Own rate of interest and investment rates for the capital and capacity stocks of MGESM $\mathrm{HL}_{\text {. }}$ Triangles denote the macroeconomic capital stocks, circles capital stocks related to energy type 1, squares capital stocks related to energy type 2 and $F$ and $R$ denote fossil and renewable based energy technologies, respectively

is less than $r^{*}$, no investor will demand credits to carry out such investment. Therefore, in capital market equilibrium one of the following two conditions for all $i^{\prime}$ holds:

$$
\begin{array}{rll}
r_{i^{\prime}}<r^{*} & \Leftrightarrow \quad I_{i^{\prime}}=0 ; \\
r_{i^{\prime}}=r^{*}>0 & \Leftrightarrow \quad I_{i^{\prime}}>0 .
\end{array}
$$

Both conditions can be summarised in a single equation:

$$
\left(r^{*}-r_{i^{\prime}}\right) I_{i^{\prime}}=0 ; \quad \forall i^{\prime}
$$

In capital market equilibrium at every point in time all ORRs of all technologies to which investments are allocated are equal and positive. Investments in each investment opportunity are increased up to the point where $r_{i^{\prime}}$ would fall below $r^{*}$. The market interest rate in turn is the result of all investment opportunities, their profitability and the preferences of the households. ORRs of all other technologies that do not attract credits are lower than $r^{*}$ and could be negative. This implies that for two alternative investment projects producing the same good three outcomes are possible: investment in both alternatives, investment in one alternative or no investment at all; see e.g. Pitchford (1979).

Thus, ORR are directly related to the investment rates (IR) of the corresponding capital and capacity stocks, because investments into a capital or capacity stock require a minimum ORR.

Figure 5 illustrates ORR and IR for the hard-link approach. Figure 5a shows the ORR for all six capital and capacity stocks in the model. The ORR of the macroeconomic capital stocks are computed by dividing the shadow price of the capital motion Eq. 14 by the shadow price of the budget Eq. 13, which results 
(a)

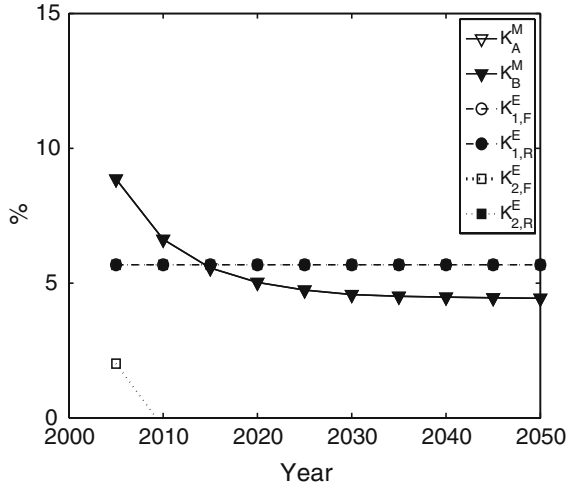

Own rate of return (b)

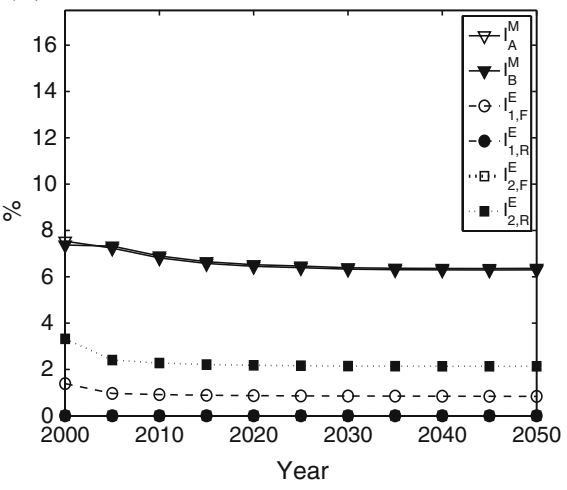

Investment rates

Fig. 6 Own rate of interest and investment rates for the capital and capacity stocks of the soft-link approach

in ORR present values for each period. The ORR of the energy capacities also require that this value is divided by the investment costs $\iota_{i, j}$.

The model calibration for the initial period indicates that capital is relatively scarce and therefore the interest rate is high. The ORRs are equal for all capital stocks in which financial means are invested. As can be seen in Fig. 5b investments $I_{2, F}^{E}$ are positive and become zero and therefore the ORR becomes lower relative to other investment opportunities. There are no investments at all in $I_{1, R}^{E}$; the corresponding ORR is negative.

The initial scarcity of capital is reduced by relatively high investment rates that are reduced in subsequent periods. Decreasing capital scarcity leads to lower ORRs. After about 25 years the market interest rate and IRs approach constant levels. The process towards the steady-state is known as transition dynamics. Note that ORR of the energy related capacity stocks is in correspondence with the ORR of the macroeconomic capital stocks. This means that all ORR of profitable investment opportunities follow along the same time path. ${ }^{2}$ This feature not only holds for the steady-state, but also for the transition dynamics.

Figure 6 shows the corresponding results for the soft-link approach. Figure $6 \mathrm{a}$ presents the ORRs of the capital and capacity stocks. ORR of the two macroeconomic capital stocks exhibit the same shape as in the hard-link, but those of $K_{1, F}^{E}$ and $K_{2, R}^{E}$ are at constant levels over the entire time horizon. This is in accordance to the exogenously assumed constant interest rate $r$ that is used in the model $\mathcal{E}^{3}$. These two capacity stocks are those to which energy related

\footnotetext{
2 This cannot be seen from the graph because the time series lie above each other, but we assure that the result is like that.

3 The constant ORR level is at about $5.7 \%$, which is slightly higher than the exogenously assumed discount rate $r$ that equals $5 \%$. The reason for this difference is not clear.
} 

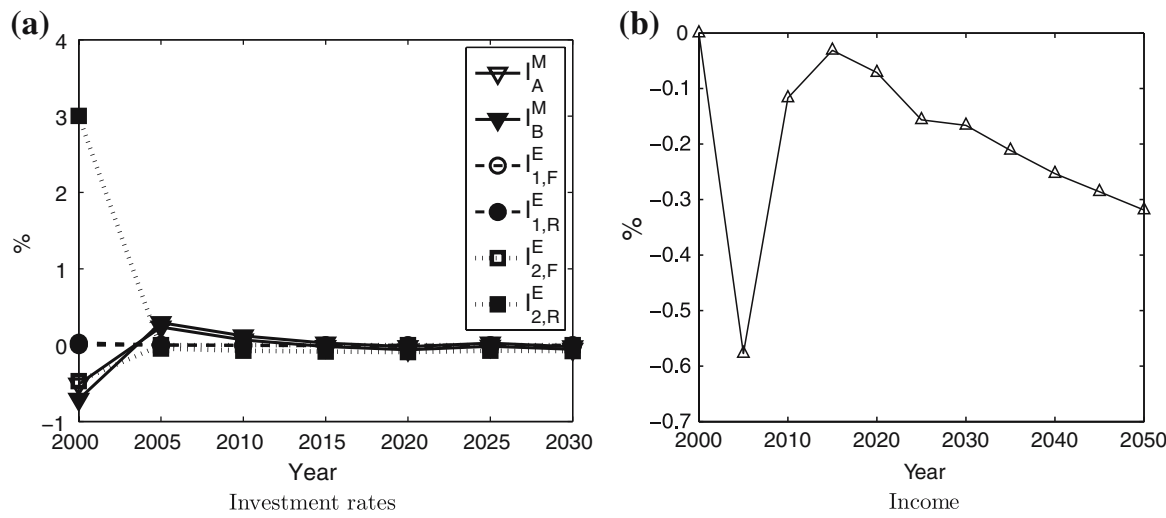

Fig. 7 Relative differences of investment rates and economic income of soft-link relative to hardlink

investments are allocated as can be seen in Fig. 6b. The ORR initially below the level of the macro-economic capital stocks induces larger energy related investments than in the hard-link approach. Thus, the differences in investments arise from a capital market dis-equilibrium in the soft-link approach that is due to the energy supply functions, which do not take scarcity of capital into account in the same way as the hard-link approach does. The energy supply functions initially signal a lower price of energy since the interest rates are initially lower and therefore the energy demand is higher. In later periods the market interest rate determined with $\mathcal{M}_{S L}$ is lower and therefore the energy investments in the soft-link are lower.

A comparison of energy capacity related investment rates in early periods reveals that the soft-link computes higher investment rates than the hard-link. This is in correspondence with the higher energy production and lower energy prices observed in Fig. 4.

A closer look at the investment dynamics is given in Fig. 7a. This graph shows the differences of the IRs by subtracting the IR of the hard-link from the soft-link's IR. It shows that in the initial period $I_{2, R}$ is $3 \%$ higher than in the hard-link. Also the investments in $I_{1, F}^{M}$ are higher in the hard-link, but since the share of $E_{1}$ is much lower than the share of $E_{2}$, the differences in the investment rates are considerably lower.

The higher investments $I_{2, R}^{E}$ are partially financed by lower investments in macroeconomic capital and energy capacities. The total initial investments of the soft-link are higher than in the hard-link, which is financed by reduced consumption. The lower initial investments in macroeconomic capital in the soft-link have to make up leeway in subsequent periods, which is the reason for the positive difference in the following two periods.

Figure $7 \mathrm{~b}$ shows the overall effect on economic income as the difference of the soft-link relative to the hard-link. It turns out that the long run economic income is about $0.3 \%$ lower than in the hard-link approach. 
In summary, the energy prices and quantities in both approaches behave quite differently. The reason is that both approaches deal differently with the intertemporal valuation of capital scarcity, which is most pronounced in early periods. The soft-link approach using the energy supply functions leads to an inconsistency at the capital market, which induces mis-allocation of investments. The overall effect on economic output is permanently negative. Therefore, the capital market dis-equilibrium induces a bias in investments reducing economic output.

\section{Extending the soft-link approach}

The inconsistency of the soft-link is related to the assumed fixed interest rate in $\mathcal{E}$. Since the MGM computes the interest rate endogenously in a consistent way, it is a natural extension of the soft-link approach to pass this information to the ESM. Therefore, we augment the mappings Eqs. 26 and 27:

$$
\begin{aligned}
& \left(\begin{array}{l}
a \\
b
\end{array}\right)=\mathcal{E}(\bar{E}, r) ; \\
& \left(\begin{array}{l}
\bar{E} \\
r
\end{array}\right)=\mathcal{M}_{S L}(a, b) .
\end{aligned}
$$

This means that the ESM considers the time varying interest rate $r$ in Eq. 10 from the MGM and summarises this additional information in the parameters $a$ and $b$. The question is whether the augmented soft-link approach solves the capital market inconsistency. ${ }^{4}$

Figure 8 shows the relative differences of energy quantities and prices of the extended soft-link approach relative to the hard-link approach. The relative differences of energy quantities in Fig. 8a shows the converse result as did the original soft-link approach. Now, the near-term energy production is lower in the soft-link approach, which leads to higher energy prices shown in Fig. 8b. The lower long-term energy production comes with higher energy prices. This suggests that the interest rate does not correctly translate into the supply function parameters and therefore the price signals in the MGM remain biased, even though the direction is converse.

From this we can conclude that the attempt to repair the defect of the soft-link has not been successful. The hard-link approach, which considers all opportunities and constraints in a single optimisation problem, leads to a consistent result. The result could not be reconciled using a static reduced form model of the dynamic full scale model $\mathcal{E}$.

4 The advantages and shortcomings discussed in Sect. 4.1 remain unchanged. 

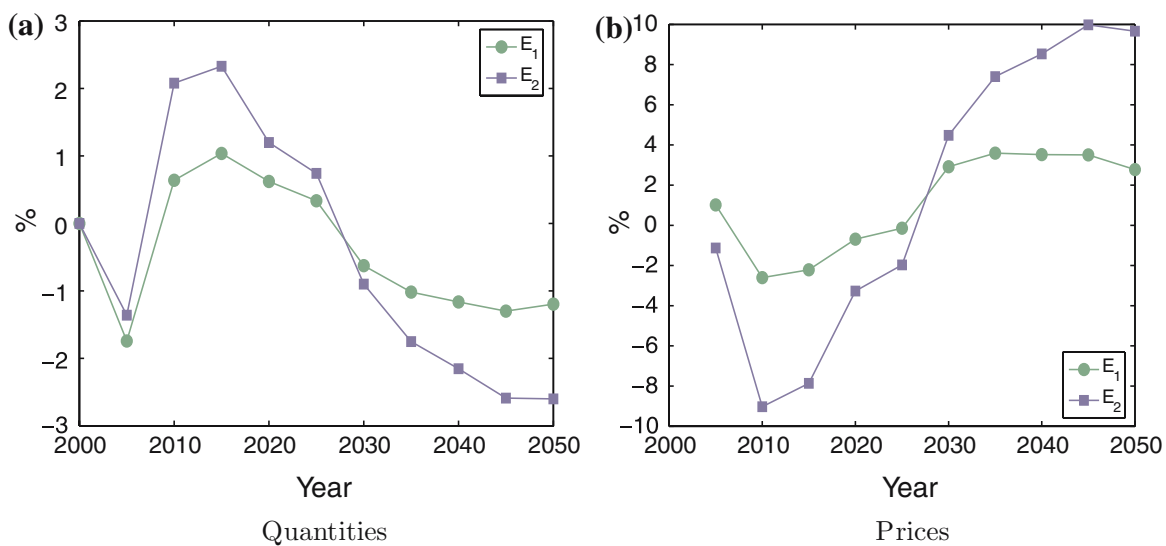

Fig. 8 Differences of the augmented soft-link relative to the hard-link approach for energy quantities and prices

\section{Discussion and conclusions}

In this study we analysed the soft and the hard-link approach for coupling models of the macroeconomy and the energy sector. It lead us to fundamental issues of production and supply theory as well as capital market equilibrium of growing economies. The analysis shows that during transition phases towards the steady-state ignoring the endogenous scarcity of capital in a supply curve induces short-run biases of investments in the ESM. The bias in energy production increases with the distance of the economy from the steady state; i.e. the effect is most important for developing countries with low per-capita income and capital stock. Moreover, as analysed in Bauer (2005, Chap. 4) and Bauer and Edenhofer (2006), the imposition of climate mitigation policies can have impacts on the interest rate, which is reduced by the availability of climate friendly technologies. A reduction of the interest rate improves the competitiveness of investment opportunities with a high cost share of capital, like renewable energy technologies. The impact of interest rate changes on the ranking of technologies increases with the differences of capital cost shares of various technologies.

The study showed that investments in energy technologies not only depend on technology parameters, resource availability and cost coefficients, but also on the macroeconomic interest rate. One issue that is not considered in this study is the impact of the wage rate development on the growth of the energy sector and technology choice therein. The experience gathered from economic time series suggests an increasing wage rate for most countries. The growth of energy production will depend on labour productivity growth in the overall economy and in the energy sector as well as in the corresponding investment good sectors. Moreover, since the ratio of wage and interest rate as well as technological progress varies across sectors and technologies, the 
relative competitiveness of investment opportunities across all sectors is permanently re-evaluated and hence technology choice is adjusted in accordance. This issue requires more research in modelling disaggregate labour in growth models.

From the present study we conclude that sound coupling of ESM and MGM requires a hard-link approach. The soft-link approach does not guarantee simultaneous equilibrium at the energy and capital market. However, this strategic choice of modelling limits the level of detail and complexity of a particular ESM. Some modelling experience is already available in the scientific literature. Bauer (2005) as well as Gerlagh and van der Zwaan (2006) integrate carbon capture and sequestration technologies. Kypreos and Bahn (2003) and Bosetti et al. (2006) integrate various types of renewable and exhaustible primary and end-use energy as well as conversion technologies.

Prospects for further inclusion of energy types and technologies in the hardlink are good. However, integration of a more detailed ESM into an MGM using the hard-link leads to new problems for example in the electricity sector: at some level of disaggregation, it is reasonable to differentiate between gas turbines and gas combined cycles. In a model that does not differentiate in peak and base load electricity the gas turbines would be uncompetitive and fade out of the system. The problem could be solved by either integrating a load curve or by introducing a bound on the minimum production share of gas turbines. The latter alternative does not increase the complexity of the model. It would make an uncompetitive investment opportunity profitable. Unfortunately, this in turn is a capital market distortion. Hence, the alternative with a higher system complexity differentiating electricity by loads would be theoretically more sound, but increases the computational difficulty significantly.

The issue of including energy technologies and the implied differentiation of energy types still requires more research. It needs to be clarified which technologies and energy types should be represented and what criteria should be applied to justify such judgements.

The advantage of the soft-link approach is that more system complexities could be represented than in the hard-link; i.e. it could satisfy stronger criteria and therefore increase the credibility of the model. It is not clear what the significance of this effect compared with the capital market distortion is and how they are interrelated. The present study does not allow a final judgement on the issue whether the soft-link should be discarded, although we revealed the flaw of the soft-link that the capital market dis-equilibrium induces misallocation of investments.

Acknowledgments The financial support of the Volkswagen-foundation is gratefully acknowledged. The support of the Swiss NCCR-Climate project, funded by the Swiss National Science Foundation (SNSF), is gratefully acknowledged. This paper is part of the research work being carried out by the Climate Change Modelling and Policy Research Program at the Fondazione Eni Enrico Mattei. 


\section{References}

Arrow KJ, Chenery HB, Minhas BS, Solow RM (1961) Capital-labor substitution and economic efficiency. Rev Econ Stat 43(3):225-250

Bauer N (2005) Carbon Capture and Sequestration - an Option to Buy Time? Phd Thesis, University Potsdam. Available online: http://opus.kobv.de/ubp/volltexte/2006/654/pdf/bauer

Bauer N, Edenhofer O (2006) Macroeconomic effects of carbon capture and sequestration within a long-term transition towards a low-carbon society. In: $8^{\text {th }}$ International Conference on Greenhouse Gas Technologies, Trondheim, Norway, accepted for publication

Boehringer C (1998) The synthesis of bottom-up and top-down in energy policy modelling. Energy Econ 20(3):233-248

Bosetti V, Carraro C, Galeotti M, Massetti E, Tavoni M (2006) WITCH: a world induced technical change hybrid model. Energy J (in press)

Dorfman R, Samuelson PA, Solow R (1958) Linear programming and economic analysis. Dover Books on Mathematics. McGraw-Hill, New York

Drouet L, Haurie A, Labriete M, Thalmann P, Vielle M, Viguier L (2006) A coupled bottomup/top-down model for GHG abatement scenarios in the Swiss housing sector. In: Loulou R, Waaub J-P, Zaccour G (eds) Energy and environment. Kluwer, Dordrecht

Edenhofer O, Bauer N, Kriegler E (2005) The impact of technological change on climate protection and welfare: insights from the model MIND. Ecol Econ 54(2-3):277-292

Frondel M, Schmidt CM (2004) Facing the truth about separability: Nothing works without energy. Ecol Econ 51(3-4):217-223

Gerlagh R, van der Zwaan BCC (2006) Options and instruments for a deep cut in $\mathrm{CO}_{2}$ emissions: Carbon capture or renewables, taxes or subsidies. Energy J 27(3):25-48

Kypreos S, Bahn O (2003) A MERGE model with endogenous technological learning. Environ Model Assess 8:249-259

Manne A, Mendelsohn R, Richels R (1995) MERGE: a model for evaluating regional and global effects of GHG reduction policies. Energy Policy 23(1):17-34

Messner S, Schrattenholzer L (2000) MESSAGE-Macro: linking an energy supply model with a macroeconomic module and solving it iteratively. Energy 25(3):267-282

Pitchford JD (1979) Two state variable problems. In: Pitchford JD, Stephen JT (eds) Application of control theory to economic analysis, Vol 101 of contributions to economic analysis, 2nd edn. North-Holland Publishing Company, Amsterdam, pp 127-154

Rafaj P (2005) Analysis of policies contributing to sustainability of the global energy system using the Global Multi-Regional MARKAL Model (GMM). Thesis Number 16122, ETH Zuerich, Switzerland

Smekens K, van der Zwaan BCC (2006) Atmospheric and geological $\mathrm{CO}_{2}$ damage costs in energy scenarios. Environ Sci Policy 9:221-227

van der Zwaan BCC, Gerlagh R, Klaassen G, Schrattenholzer L (2001) Endogenous technological change in climate change modelling. Energy Econ 24:1-19

Wing IS (2006) The synthesis of bottom-up and top-down approaches to climate policy modeling: electric power technologies and the costs limiting us $\mathrm{CO}_{2}$ emissions. Energy Policy 34(18):38473869 